\title{
SURGICAL SITE INFECTION RELATED RISK FACTORS AND USAGE OF ANTIBIOTICS IN TWO DIFFERENT TERTIARY CARE HOSPITALS OF DHAKA CITY, BANGLADESH
}

\author{
FORHAD MONJUR ${ }^{1 *}$, FARHANA RIZWAN ${ }^{2}$, NOBO KRISHNA GHOSH ${ }^{3}$
}

${ }^{1}$ Department of Clinical Pathology, Institute of Child Health \& Shishu Sasthya Foundation Hospital, 6/2, Barabagh, Mirpur-2, Dhaka-1216, Bangladesh. ${ }^{2}$ Department of Pharmaceutical Technology, Jadavpur University, Kolkata-32, India \& Department of Pharmacy, East West University, Aftabnagar, Dhaka, Bangladesh. ${ }^{3}$ Department of Paediatric Medicine, Institute of Child Health \& Shishu Sasthya Foundation Hospital, 6/2, Barabagh, Mirpur-2, Dhaka-1216, Bangladesh. Email: monjur_forhad@yahoo.com

Received: 19 March 2018, Revised and Accepted: 25 April 2018

\section{ABSTRACT}

Objective: Surgical site infection (SSI) is the most common complications following surgical procedures. The aim of the study was to assess the risk factors for SSI and the antibiotic treatment pattern following post-operative infection.

Methods: This retrospective study has been considered the data of 156 surgical patients from two tertiary care hospitals in Dhaka city of Bangladesh.

Result: The majority (31.4\%) of the surgical patients came from the age group 40 to 49 years. A total of 8 SSI cases were discovered, 5 cases (8.9\%) from Hospital-1, which was relatively higher (3\%) than Hospital-2. Wound class "contaminated" was found in 6 cases, and the ratio is almost $1: 3$ or $75 \%$. The duration of surgery within $90-120 \mathrm{~min}$ for $50 \%$ of SSI patients (4/8). Most frequent types of surgery were colorectal surgery (56.4\%, $88 / 156)$, followed by various grades of fistula (24.4\%, 38/156). The prophylaxis antibiotic was ceftriaxone, used by 77 (49.4\%) cases. Commonly used antibiotic was ciprofloxacin, administered in 33 (21.2\%) patients as a single therapy. Third-generation cephalosporins (cefixime, ceftriaxone, and cefuroxime) were found at the drug of choice in combination with gentamicin/metronidazole/nitazoxanide or amikacin.

Conclusion: This study tried to give a little overview of SSI in different hospital settings, whereas improvement of preventive measures, early detection, and management of SSI is the main concern of nowadays. Further research should be done to get the detail by considering all the relevant factors of SSI.

Keywords: Surgical site infection, Prophylaxis antibiotics, Post-operative antibiotics.

(C) 2018 The Authors. Published by Innovare Academic Sciences Pvt Ltd. This is an open access article under the CC BY license (http://creativecommons. org/licenses/by/4. 0/) DOI: http://dx.doi.org/10.22159/ajpcr.2018.v11i8.25878

\section{INTRODUCTION}

Surgical site infection (SSI) is the most frequent type of nosocomial infections following surgical procedures. SSI is associated with delayed recovery, prolonged hospitalization, and increased incidence of mortality and morbidity [1-4]. The Centers for Disease Control and Prevention guideline for the prevention of SSI, established the criteria for SSI, was published in 1999 and reported the post-operative infection as the second most common type (22\%) of healthcare-associated infection [1].

Antibiotics have the potential impact on SSI to prevent mortality [5]. Over the years, the principles of surgical prophylaxis have been established by various countries. Accurate antibiotic therapy reduces the incidence of SSIs. Besides, inappropriate and unnecessary use of antibacterial agents can contribute to bacterial resistance and increase the economic burden to patients' family and society also $[2,3,5,6]$.

The incidence of SSI varies widely, and it was $<1 \%$ to $>10 \%$ in different parts of the world $[3,5,7]$. Tanzania reported that the SSI rate was $19.4 \%$ [5]. In the United States of America (USA), approximately one million patients develop SSI of varying severity each year [5]. In 1999, after surgery, SSI increased the patients' risk of death (from 3.5\% to $7.8 \%$ ), increased the risk of ICU stay (from 18\% to 29\%), added 5 days to the hospital stay, doubled the cost of hospitalization, and increased the possibility of readmission (from $7 \%$ to $41 \%$ ), observed in another report of the USA [8].

SSI significantly interferes the potential benefits of post-operative patients [4]. Thus, it is very vital to assess the responsible factors of SSI and take the necessary measures to prevent the occurrence of nosocomial infection and to reduce the morbidity and mortality and their medical expenses as well.

\section{METHODS}

\section{Study design}

The retrospective study was carried out from January to December 2014. 156 data of post-operative patients were reflected in the present study. Patient's previous data had been taken which include diagnosis and treatment that the patient had undergone. Gonosasthaya Nagar Somajvittik Hospital, Dhaka (Hospital-1), and Japan-Bangladesh Friendship Hospital, Dhaka (Hospital-2), are the tertiary care hospitals and were chosen for the study area.

\section{Statistical analysis}

The statistical analysis was performed with Microsoft Excel 2016.

\section{RESULTS}

\section{Sample distribution}

The post-operative patients were $35.9 \%(56 / 156)$ from Hospital- 1 and 64.1\% (100/156) from Hospital-2. Among them, 80.12\% (125/156) patients were male. The mean age of the total patient was 42.68 years. Most of (the cases (31.4\%) came from the age group 40-49 years.

\section{Disease pattern of study populations}

Most common surgical types were colorectal surgery followed by various grades of fistula $(24.4 \%, 38 / 156)$. Various degrees of hemorrhoids (two, three, and four degrees) were discovered in $14.1 \%$ (22/156) of cases. Abdominal infections such as appendicitis and open cholecystectomy 
were common in $22(14.1 \%)$ cases. Patients with different grades of hernia were found in 14 (9\%) cases. Other diseases including multiple fibroids, cholelithiasis, hemangioma, breast carcinoma, and maxillary sinusitis were 18.6\% (20/156) (Fig. 1)

\section{The pre-operative antibiotic usage pattern}

Most frequently used prophylaxis antibiotics were ceftriaxone (49\%, $77 / 156)$, ciprofloxacin (22\%), and cefuroxime (9\%). Again, ciprofloxacin and metronidazole $(5.1 \%, 8 / 156)$ were given as combination antibiotic therapy also (Fig. 2).

\section{The post-operative antibiotic usage pattern}

Ciprofloxacin $(21.2 \%, 33 / 156)$ was given to post-operative patients as a sole therapy in maximum cases. It was also used in combination with metronidazole (6.4\%) and ceftriaxone (5.8\%). Other frequently used antibiotics were ceftriaxone $(21,13.5 \%)$. Third-generation cephalosporins were in top notches such as ceftriaxone (13.5\%), cefuroxime $(6.4 \%)$, and combination with gentamicin, metronidazole, nitazoxanide, and amikacin. At Hospital-1, the most commonly used antibiotic was ciprofloxacin (Fig. 3)

\section{Post-operative antibiotic combination}

In most of the post-operative cases, combination antibiotic therapy was preferable. A combination of two antibiotics was the most in $74(47.4 \%)$ cases and combination of three antibiotics in 12 (7.7\%) cases (Fig. 4).

\section{SSI rate in both hospitals}

At Hospital-2, only 3\% (3/100), and Hospital-1, 8.92\% (5/56) SSI cases were found among the post-operative patients.

\section{Wound class factor and SSI}

Depending on the US National Research Council group classification, the wound class cases were found to have clean $(55 / 156, \mathrm{SSI}=1)$, clean contaminated $(2 / 156, \mathrm{SSI}=1)$, and contaminated $(99 / 156, \mathrm{SSI}=6)$ in this study. Here, $75 \%$ of SSI patients were discovered from the contaminated group (Fig. 5).

\section{Diabetic patients and SSI}

Of 156 patients, 35 had diabetes mellitus, and of them, 6 had developed SSIs. From 121 non-diabetic patients, only 2 had developed by SSI.

\section{American Society of Anesthesiologist (ASA) classification system} and SSI

In 1963, ASA adopted the 5-category physical status classification system based on the fitness of patients before surgery; a sixth $\left(6^{\text {th }}\right)$

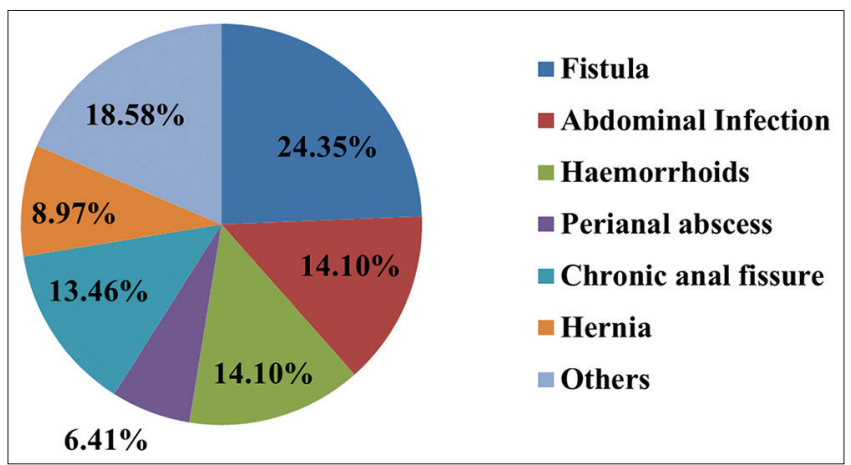

Fig. 1: Disease pattern of the operative cases category was later added. In this study, category 1 had one, category 2 had three, and category 3 had four SSI cases. No cases were found in category 4 or 5 (Fig. 6)

\section{Duration of surgery and hospital stay}

Of 8 SSI cases, $50 \%(4 / 8)$ of patients had the duration of surgery within 90-120 min. However, duration of surgery within 60-80 minutes was found in 2 (25\%) cases only. Apart from this, no SSI patient has been found operating within a short course of time (10 to $20 \mathrm{~min}$ ). Duration of staying at the hospital was discovered for 3 days (35.9\%) and 4 days (17.9\%), respectively. In about $0.6 \%$ of cases, patients had to stay for 22 days at Hospital-1, which was larger than Hospital-2 (Table 1).

\section{DISCUSSION}

SSI remains the most common surgical complications. The rates of SSI are increasing globally even in hospitals with the most modern facilities [3]. The most important risk factors for SSI are the type and duration of surgery, operative technique, surgeon's skill, and pre-operative preparation of the surgical site. Management of these problems has contributed to the overall decline in the rate of SSI [9].

In this current study, $31.4 \%$ of cases came from the age group 4049 years. A similar study conducted in Pune, India, had $32.5 \%$ operative patients lying under the age group 34-49 years [10], whereas the majority of the surgical patients were between the ages 18-40 years which were found in a separate study of India [11]

The rate of incidence of SSI does not alike for all the hospitals, and it differs widely from one hospital to another also. In China, the rate of SSI varies from $13.0 \%$ to $18.0 \%$ [3]. A huge number of studies reported the SSI cases $(3.34 \%, 7.3 \%, 1.5 \%$, and $4.4 \%)$ in surgical patients $[3,5,12,13]$. All these findings showed similarities with the current study, 5.1\% $(8 / 156)$. Furthermore, Paarakh et al. found that the rate of SSI was $48.3 \%$ $(68 / 120)$ in their study [14], which is quite higher than the other findings.

Prophylaxis antibiotics inhibit the growth of bacteria and thus reduce the possibility of infection. Some clinical trials have been proposed by the association. Although a large number of studies did not find any co-relation between the starting time of prophylaxis antibiotic and the occurrence of SSI; it was reported by some studies that antibiotic prophylaxis have no role in the occurrence of SSI and prevention of infection, even administered for the longer duration $[1,15]$. However, the lowest rate of SSI was found in those cases where the antibiotics started 2 hours before an operation, mentioned in a different study [12]. Whereas, higher rates of SSI in whom antimicrobial prophylaxis were started $12 \mathrm{~h}$ before surgery which have been observed by Stone et al. [16].

The antibiotic usage pattern of the current study supports the findings of Bishnu et al. [5]. The most commonly prescribed antibiotics were ceftriaxone (38\%) as reported by Joshi et al. [11]. Besides, top postoperative antibiotics prescribed were metronidazole, cefotaxime, and ceftriaxone which were found in two different studies in India $[6,10]$. Amoxicillin (26\%) was discovered as a drug of choice, followed by penicillin (20\%) [14], whereas the most common antimicrobial agents used were cefotaxime (81\%), followed by cefoperazone sodium (42\%), ceftriaxone $(25 \%)$, and amoxicillin with clavulanate $(20 \%)$ in surgical patients [17].

Combination antibiotic therapy was administered for synergistic action and to cover the broad spectrum of microorganisms in post-operative

Table 1: Duration of surgery and SSI

\begin{tabular}{lllllll}
\hline SSI & Duration of operation & \multicolumn{1}{c}{ Total } \\
\cline { 2 - 6 } & Different variables & $\mathbf{3 0 - 5 0} \mathbf{~ m i n}$ & $\mathbf{6 0 - 8 0} \mathbf{~ m i n}$ & $\mathbf{1 0 - 2 0} \mathbf{~ m i n}$ & $\mathbf{9 0 - 1 2 0} \mathbf{m i n}$ & $\mathbf{> 1 2 0}$ min \\
\hline Total SSI cases (N=8) & 1 & 2 & 0 & 4 & 1 & 8 \\
\% within SSI & 12.5 & 25.0 & $0.0 \%$ & 50.0 & 12.5 & 100.0 \\
\hline
\end{tabular}

Here, total number of SSI cases, N=8; total number of patients=156. SSI: Surgical site infection 


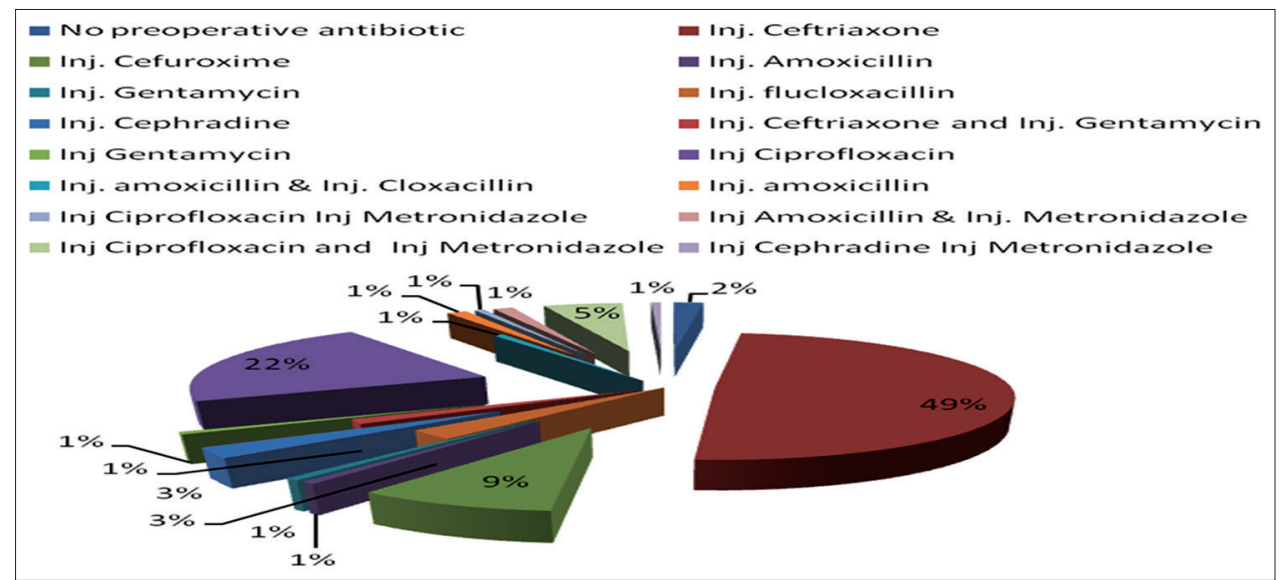

Fig. 2: Pre-operative antibiotic usage pattern

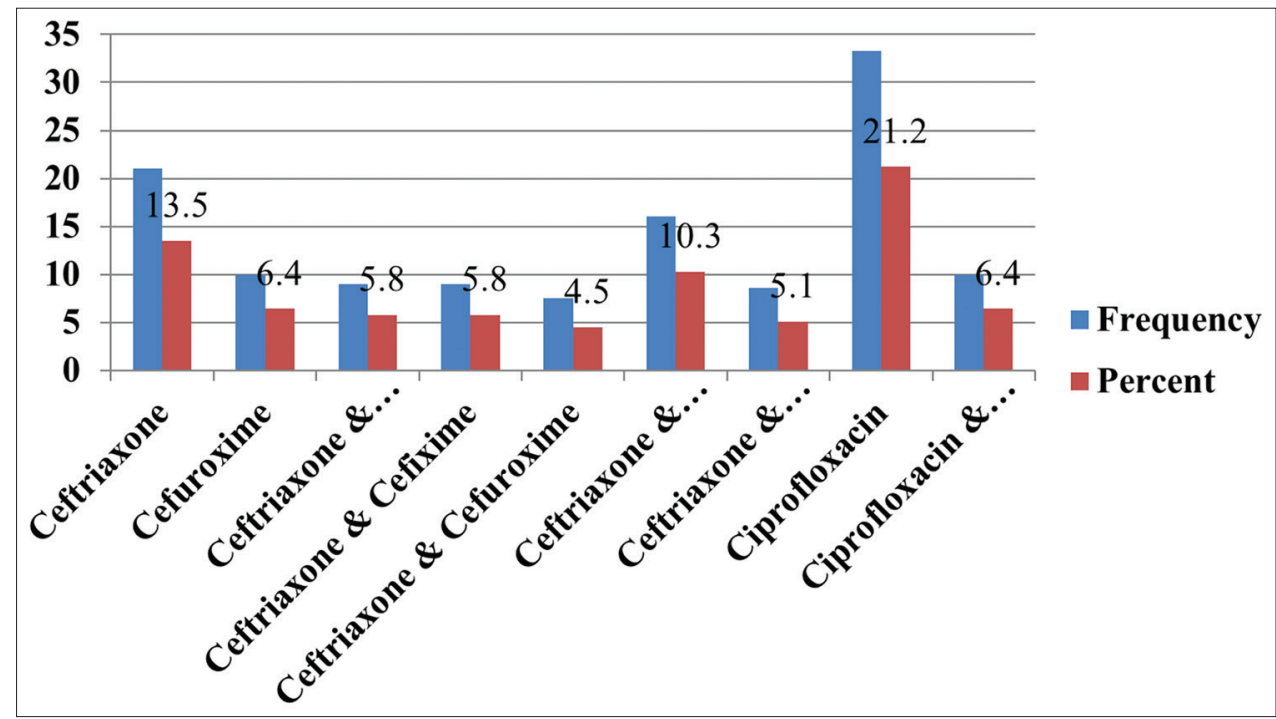

Fig. 3: Post-operative antibiotic usage pattern

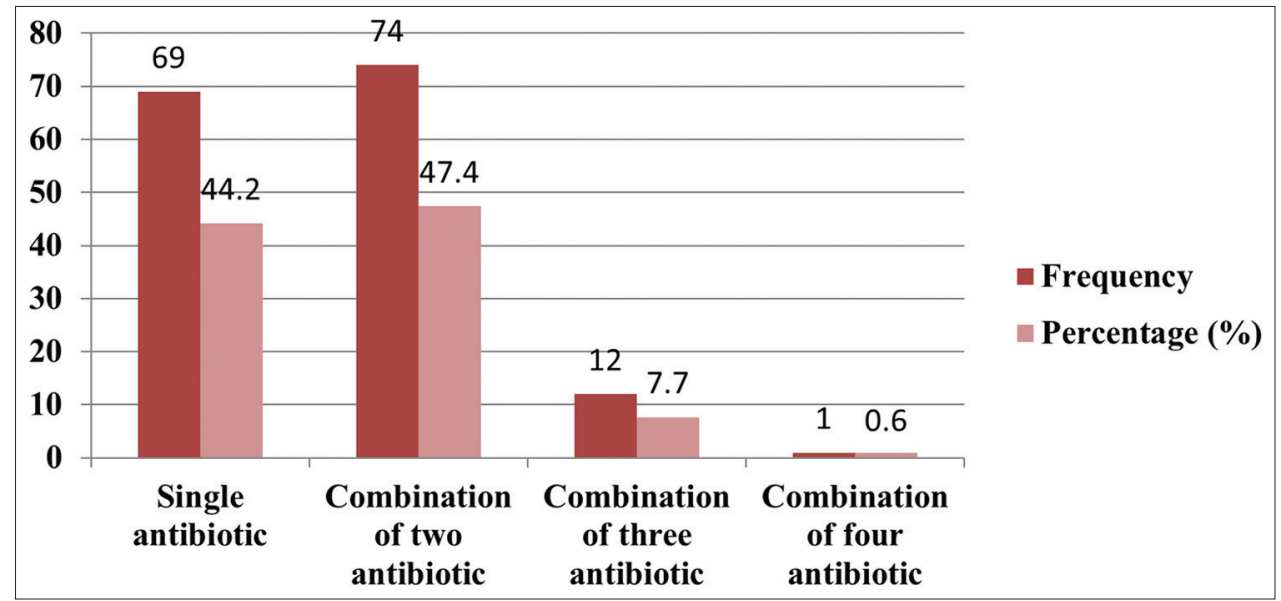

Fig. 4: Combination of antibiotic usage pattern

cases. In the current study, a combination of two antibiotics was $47.4 \%$ $(74 / 156)$ and the combination of three antibiotics was $7.7 \%(12 / 156)$. The antibiotic combination of cefotaxime with metronidazole $(35 \%)$ followed by ceftriaxone (25.3\%) and ampicillin $(17.2 \%)$ was found in a separate study [18]. Besides, $39 \%$ of patients received antibiotic combination and $18 \%$ of patients received monotherapy of antibiotic [11].
The maximum SSI cases were found in the contaminated group $6 / 8$, $75 \%)$ in this study, whereas the contaminated (8\%) and dirty groups (14.8\%) of wound classes were discovered in Nepal [5]. However, another study conducted in Bangladesh reported that the majority of SSI cases were coming from dirty group (52\%), followed by contaminated (32\%) group of wound classes [19]. 


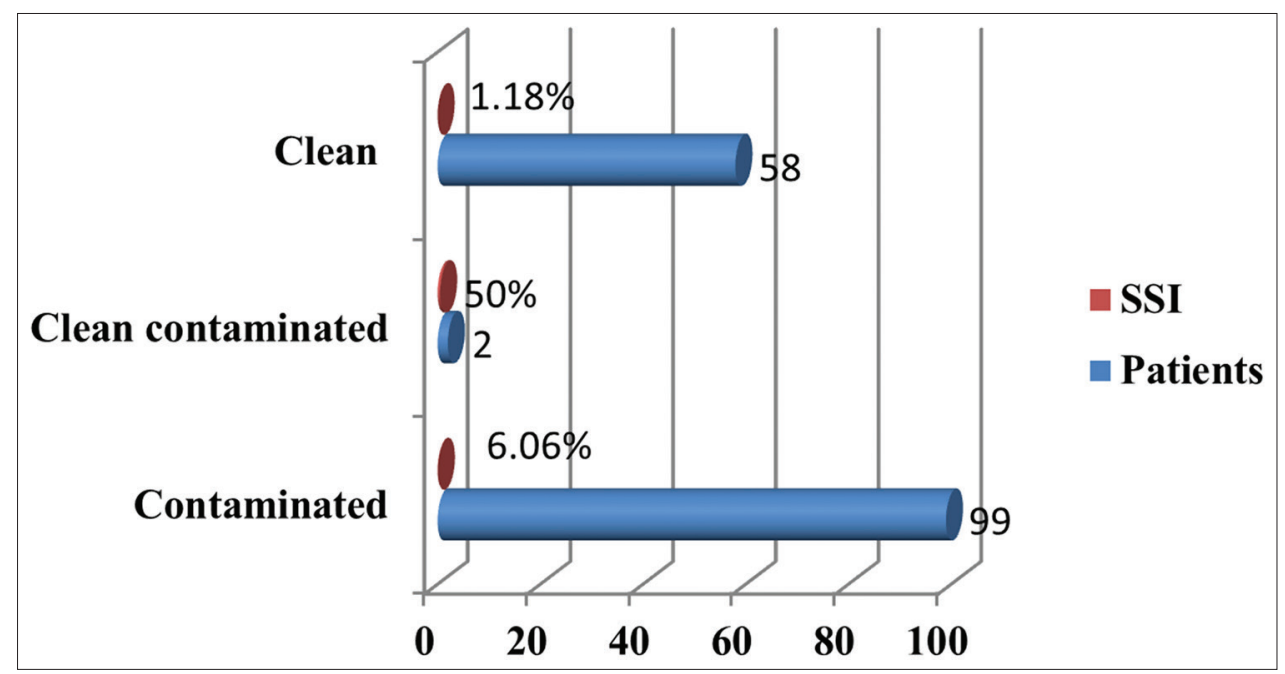

Fig. 5: Wound class factor and surgical site infection

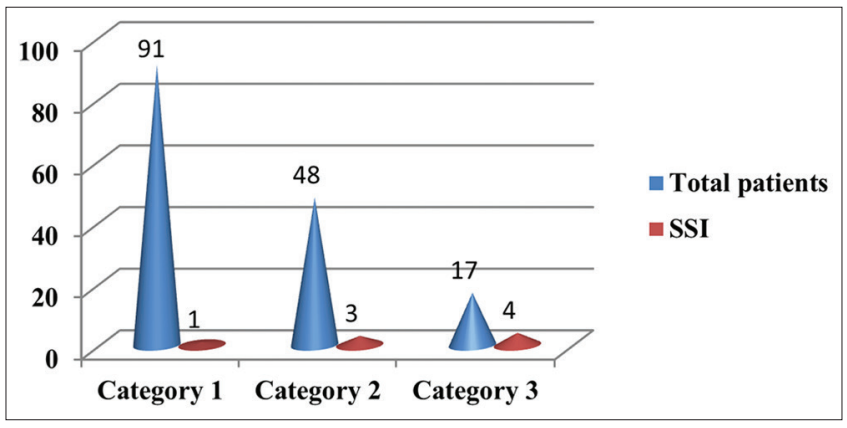

Fig. 6: American Society of Anesthesiologist physical status classification system and surgical site infection

The clinical relevance was found for the patients, who were suffering from a serious pre-existing disease (diabetes mellitus) from various studies. They also have a higher risk of developing SSI, and there was a significant difference between the diabetic and non-diabetic patient groups [3,5]. In the current study, 6 cases developed SSI, of 35 diabetic patients. From 121 non-diabetic patients, only 2 cases developed SSI. This finding showed the similarity with another study conducted by Reiping et al. [1].

A variable number of studies showed the existence of the exact relationship between the operative time and the incidence of SSI [20,21]. The SSI rate found $6.3 \%$ for $1 \mathrm{~h}, 12.2 \%$ in $1-2 \mathrm{~h}$, and $27.7 \%$ for more than $2 \mathrm{~h}$ in a study [22]. If the operative duration is $>2 \mathrm{~h}$, then there is a higher chance of SSI, suggested by Keping et al. and the current study [3]. Some literature shows the relationship between the incidence of SSI and the duration of hospital stay. The current study revealed the maximum duration of staying ( 22 days) at the hospital and the incidence of SSI $(0.6 \%)$. Besides, a comparative study between two hospital settings reported the greater severity of SSI with longer duration of hospital stay [23], whereas $48 \%$ of SSI patients stayed at the hospital for more than 5 days as reported by Joshi et al. [11].

This study has been conducted based on the previous hospital record and also done in a small number of populations. Records from the general surgery department were enrolled in this particular research. Hence, further research should be done considering the particular department. Our study concluded with the risk factor that may help to lower the prevalence of SSI and the antibiotic usage pattern for postoperative patients. Again, only the data for 1 year were not enough to make any concrete decision and are not necessarily representative of the other year.

\section{ACKNOWLEDGMENT}

The authors would like to acknowledge all the administrative staffs and doctors of Gonosasthya Nagar Somajvittik Hospitals (Hospital-1), Dhaka, and Japan-Bangladesh Friendship Hospital (Hospital-2), Dhaka, for their continuous support and technical assistance to conduct the study.

\section{AUTHORS' CONTRIBUTIONS}

Dr. Forhad Monjur has been involved in the development of concepts, design, the definition of intellectual content, data analysis, statistical analysis, manuscript editing, manuscript review, and guarantor. Farhana Rizwan has been implicated in the development of concepts, design, literature search, data acquisition, data analysis, manuscript preparation, and manuscript review. Dr. Nobo Krishna Ghosh has been designed the concepts, the definition of intellectual content, manuscript preparation, manuscript editing, and manuscript review.

\section{CONFLICTS OF INTEREST}

The authors declare that there are no conflicts of interest.

\section{REFERENCES}

1. Tang R, Chen HH, Wang YL, Changchien CR, Chen JS, Hsu KC, et al. Risk factors for surgical site infection after elective resection of the colon and rectum: A single-center prospective study of 2,809 consecutive patients. Ann Surg 2001;234:181-9.

2. Basavaraj B, Satyanarayana V, Jyotirmoy A. A retrospective study of the pattern of antimicrobial prophylaxis and surgical site infection in abdominal surgeries in a tertiary care hospital. Natl J Basic Med Sci 2013;3:221-6.

3. Cheng K, Li J, Kong Q, Wang C, Ye N, Xia G, et al. Risk factors for surgical site infection in a teaching hospital: A prospective study of 1,138 patients. Patient Prefer Adherence 2015:9:1171-7.

4. Song L, Ji M, Gefei W, Meng W, Xiuwen W, Kun G, et al. Risk factors for postoperative surgical site infections in patients with Crohn's disease receiving definitive bowel resection. Sci Rep 2017;7:1-6.

5. Bishnu RG, Hom PP, Ravi SP, Chandrasekhara TS, Pranav KS. Surgical site infection and antibiotics use pattern in a tertiary care hospital in Nepal. J Pak Med Assoc 2008;58:141-51.

6. Rajeshwari, Nagabhusan H. Drug utilization study in postoperative patients in obstetrics and gynecology ward of a tertiary care hospital. Int J Basic Clin Pharm 2016;2016:329-4.

7. Awad SS. Adherence to surgical care improvement project measures and postoperative surgical site infections. Surg Infect (Larchment) 2012;13:234-7.

8. Kirkland KB, Briggs JP, Trivette SL, Wilkinson WE, Sexton DJ. The impact of surgical-site infections in the 1990s: Attributable mortality, excess length of hospitalization, and extra costs. Infect Control Hospital 
Epidemiol 1999;20:725-30.

9. Hunt TK. Surgical postoperative wound Infections: An overview. Am J Med 1981;70:712-8.

10. Raut A, Cherian T, Chauhan S, Pawar A. Antibiotic utilization pattern at the surgery department of a tertiary care hospital. Asian J Pharm Clin Res 2017;10:131-4.

11. Devesh KJ, Mohd R, Preeti K, Yogesh J. Evaluation of a prescription pattern of antibiotics for surgical prophylaxis in a secondary care hospital. Int J Basic Clin Pharmacol 2017;6:1969-76.

12. Classen DC, Evans RS, Pestotnik SL, Horn SD, Menlove RL, Burke JP, et al. The timing of prophylactic administration of antibiotics and the risk of surgical-wound infection. N Engl J Med 1992;326:281-6.

13. Chuang SC, Lee KT, Chang WT, Wang SN, Kuo KK, Chen JS, et al. Risk factors for wound infection after cholecystectomy. J Formos Med Assoc 2004;103:607-2.

14. MihirKumar B, Ashley S, Nawaz S, Surekha G, Padmaa MP. Assessment of two or more antibiotic regimens versus one or no antibiotic regimen in postoperative surgery. Int J Pharm Pharm Sci 2017;9:188-1.

15. Pankaj G, Manish S, Bhushan SW, Vijay MB, Dilip SG, Dhirendra DW, et al. Role of antibiotics on surgical site infection in cases of open and laparoscopic cholecystectomy: A comparative observational study. J Surg Tech Case Rep 2014;6:1-4.

16. Stone HH, Hooper CA, Kolb LD, Geheber CE, Dawkins EJ.
Antibiotic prophylaxis in gastric, biliary and colonic surgery. Ann Surg 1976; $184: 443-2$

17. Kala P, Jamuna RR, Sangeeta R. Prescription pattern of prophylactic antimicrobial agents used in preoperative patients at a tertiary care teaching hospital. Int J Pharm Pharm Sci 2018;10:128-1.

18. Heethal J. Pattern of antimicrobial use in cesarean section in a tertiary care hospital in rural south India. Int J Pharm Biomed Res 2010;1:57-61.

19. Tamanna FT, Ahmed Z. Bacteriological study of postoperative abdominal wound infection-a case study. Bangladesh J Med Sci 2013;12:86-90.

20. Cruse PJ, Foord R. The epidemiology of wound infection: A 10-year prospective study of 62939 wounds. Surg Clin North Am 1980;60:27-40.

21. Public Health Laboratory Services. Incidences of surgical wound infection in england and wales: A report of public health laboratory service. Lancet 1960;2:658.

22. Patric P, Simon M, David A, Jean-Marie H, Yvs F. Risk factors for postoperative infectious complications in non-colorectal abdominal surgery. A multivariate analysis based on a prospective multicenter study of 4718 patients. Arch Surg 2003;138:314-24.

23. John JE, Yehuda C, Sara EC, Vance GF, Melissa ZB, Sharon LT, et al. Adverse clinical and economic outcomes attributable to methicillin resistance among patients with Staphylococcus aureus surgical site infection. Clin Infect Diseases 2003;36:592-8 